Papers read at the Annual Scientific Meeting of the International Medical Society of Paraplegia held at Stoke Mandeville, Aylesbury, England, July, 198I

Session on Spinal Stenosis

\title{
REHABILITATION OF MICTURITION BY TRANSURETHRAL ELECTROSTIMULATION OF THE BLADDER IN PATIENTS WITH INCOMPLETE SPINAL CORD LESIONS
}

\author{
By H. Madersbacher, M.D., W. Pauer, M.D., and E. Reiner, M.D. \\ S. Spanudauis Rehabilitationszentrum Bad Häring, Austria.
}

\begin{abstract}
This study shows that in selected patients with incomplete spinal cord lesions, micturition may be aided by a new technique of transurethral electro stimulation.
\end{abstract}

Key Words: Spinal cord lesions; Micturition disturbance; Transurethral electrostimulation of the bladder.

SINCE the description of transurethral electrostimulation by Katona (1956) controversial opinions have been published. In contrast to other types of electrical bladder stimulation the physiological basis for this method of treatment is the stimulation of receptors in the bladder wall.

This report presents our results over a 4-year period using stimulation parameters other than those published to date and studying the effects objectively by using urodynamic techniques.

\section{Method}

Twenty nine patients with incomplete lesions of the spinal cord after injury were treated from I4 days to 8 months after injury (average 3 months). Seventeen of these patients were confined to wheelchairs and five had limited ambulation with crutches.

Stimulation was carried out by impulse packages applied to a saline (0.9I) filled bladder. Their duration, the intervals in between and their rise time-that is the time until the maximal selected current is achieved-can be varied from I to Io seconds. Each package is built up by impulses with a current varying between $\mathrm{I}$ and Io milliamps, with an impulse duration between 6 and $8 \mathrm{msec}$ and a frequency of 70 to $100 \mathrm{~Hz}$. Thus, individual stimulation can be applied according to different types of neurogenic bladder dysfunction. The scheduled treatment comprises a minimum of about 50 stimulations with a daily stimulation up to 90 minutes. The course is terminated if no bladder sensation or changes in the pattern of bladder activity occur during this period, otherwise it is continued until maximum improvement is achieved.

Before electrostimulation was started, the function of the detrusor-sphinctercomplex was evaluated by combined urodynamic studies including voiding cystourethrography. Control of therapy comprises a continuous registration of intravesical and intra-rectal pressure during stimulation as well as cystomanometry during and after treatment. If necessary, the degree of incontinence was assessed by the urilos-meter ${ }^{\mathrm{R}}$ (see Table I). 
TABLE I

Results of intravesical electrostimulation

\begin{tabular}{|c|c|c|c|c|c|c|c|c|c|c|}
\hline \multirow{2}{*}{$\begin{array}{l}\text { Name, } \\
\text { age } \\
\text { L. W. } 20\end{array}$} & \multirow{2}{*}{$\begin{array}{l}\text { Sex } \\
\text { F }\end{array}$} & Lesion & $\begin{array}{l}\text { Stim. after } \\
\text { injury }\end{array}$ & $\begin{array}{l}\mathrm{Nr} \\
\text { stim. }\end{array}$ & \multicolumn{2}{|c|}{ Sensation } & \multicolumn{2}{|c|}{ Detrusor function } & $\begin{array}{l}\text { Contro } \\
\text { before }\end{array}$ & $\begin{array}{l}\text { mictn. } \\
\text { after }\end{array}$ \\
\hline & & $\begin{array}{l}\text { Incompl. } \\
\text { Sub L4 }\end{array}$ & 3 Months & 54 & $\neq$ & + & - & + & - & + \\
\hline F. J. 20 & $M$ & $\begin{array}{l}\text { Incompl. } \\
\text { Sub L4 }\end{array}$ & 5 Months & 32 & - & + & $\neq$ & + & - & + \\
\hline M. P. 24 & M & $\begin{array}{l}\text { Incompl. } \\
\text { Sub TH7 }\end{array}$ & 2 Months & 34 & $\neq$ & + & - & + & - & + \\
\hline K. J. 54 & M & $\begin{array}{l}\text { Incompl. } \\
\text { Sub C8 }\end{array}$ & 5 Months & 27 & - & + & - & + & - & + \\
\hline M. H. 43 & $\mathrm{~F}$ & $\begin{array}{l}\text { Incompl. } \\
\text { Sub L3 }\end{array}$ & 4 Months & 63 & $\neq$ & + & - & + & - & + \\
\hline H. H. I 8 & $\mathrm{M}$ & $\begin{array}{l}\text { Incompl. } \\
\text { Sub TH4 }\end{array}$ & 8 Months & 24 & + & + & + & + & - & + \\
\hline K. J. 48 & M & $\begin{array}{l}\text { Incompl. } \\
\text { Sub C7 }\end{array}$ & 2 Months & 36 & + & + & $\neq$ & + & - & + \\
\hline A. A. 53 & $M$ & $\begin{array}{l}\text { Incompl. } \\
\text { Sub L4/5 }\end{array}$ & 3 Months & 23 & - & + & $\neq$ & + & - & + \\
\hline T. J. 39 & M & $\begin{array}{l}\text { Incompl. } \\
\text { Sub C6/7 }\end{array}$ & 3 Months & 28 & - & + & - & + & - & + \\
\hline P. H. 2 I & $M$ & $\begin{array}{l}\text { Incompl. } \\
\text { Sub SI }\end{array}$ & 3 Months & 49 & - & + & $\neq$ & + & - & - \\
\hline K. CH. 23 & M & $\begin{array}{l}\text { Incompl. } \\
\text { Sub C7 }\end{array}$ & 4 Months & 34 & + & + & - & + & - & $\neq$ \\
\hline B. E. 60 & M & $\begin{array}{l}\text { Incompl. } \\
\text { Sub THr2 }\end{array}$ & 2 Months & 132 & - & + & - & - & - & $\neq$ \\
\hline M. D. 32 & M & $\begin{array}{l}\text { Incompl. } \\
\text { Sub C6 }\end{array}$ & 4 Months & 85 & $\neq$ & + & - & + & - & + \\
\hline A. F. 48 & $\mathbf{M}$ & $\begin{array}{l}\text { Incompl. } \\
\text { Sub LI/THI2 }\end{array}$ & I Month & 90 & $\neq$ & + & $\neq$ & + & - & + \\
\hline
\end{tabular}




\begin{tabular}{|c|c|c|c|c|c|c|c|c|c|c|}
\hline W. M. 33 & $M$ & $\begin{array}{l}\text { Incompl. } \\
\text { Sub LI }\end{array}$ & 3 Months & 65 & $\neq$ & + & - & $\neq$ & - & $\neq$ \\
\hline V. J. I8 & $M$ & $\begin{array}{l}\text { Incompl. } \\
\text { Sub LI }\end{array}$ & I Month & 43 & $\neq$ & $\neq$ & - & - & - & - \\
\hline L. A. I9 & $M$ & $\begin{array}{l}\text { Incompl. } \\
\text { Sub C6/C7 }\end{array}$ & 3 Months & 65 & $\neq$ & + & - & + & - & + \\
\hline P. K. 35 & $M$ & $\begin{array}{l}\text { Incompl. } \\
\text { Sub C6/C7 }\end{array}$ & 6 Months & 53 & - & + & $\neq$ & + & - & + \\
\hline H. J. 32 & $\mathrm{M}$ & $\begin{array}{l}\text { Incompl. } \\
\text { Sub C6/C7 }\end{array}$ & 7 Months & 55 & $\neq$ & + & + & + & $\neq$ & $\neq$ \\
\hline L. M. 33 & $\mathrm{~F}$ & $\begin{array}{l}\text { Incompl. } \\
\text { Sub LI }\end{array}$ & 2 Months & 90 & - & + & - & + & - & $\neq$ \\
\hline P. F. 44 & $M$ & $\begin{array}{l}\text { Incompl. } \\
\text { C5/6-C6/7 }\end{array}$ & 4 Months & 50 & $\neq$ & + & $\neq$ & + & - & + \\
\hline S. I. I8 & $M$ & $\begin{array}{l}\text { Incompl. } \\
\text { Sub C5 }\end{array}$ & 3 Months & 29 & + & + & $\neq$ & + & - & $\neq$ \\
\hline Z. P. 27 & $\mathrm{M}$ & $\begin{array}{l}\text { Incompl. } \\
\text { Sub C5/6 }\end{array}$ & 3 Months & 9I & $\neq$ & $\neq$ & $\neq$ & $\neq$ & - & - \\
\hline L. P. 22 & $M$ & $\begin{array}{l}\text { Incompl. } \\
\text { Sub LI }\end{array}$ & I4 Days & 52 & - & $\neq$ & - & + & - & + \\
\hline U. P. 38 & $M$ & $\begin{array}{l}\text { Incompl. } \\
\text { Sub L4 }\end{array}$ & 2 Months & 74 & + & $\neq$ & - & + & - & + \\
\hline M. M. 30 & $\mathrm{M}$ & $\begin{array}{l}\text { Lamin. } \\
\text { THII-L2 }\end{array}$ & 2 Months & 42 & + & + & + & + & - & - \\
\hline L. F. 4I & $M$ & $\begin{array}{l}\text { Incompl. } \\
\text { Sub } \mathrm{TH}_{4-5}\end{array}$ & I Month & 43 & + & $\neq$ & - & + & - & + \\
\hline U. O. 46 & $\mathrm{M}$ & $\begin{array}{l}\text { Incompl. } \\
\text { Sub THI I }\end{array}$ & 3 Months & 33 & + & $\neq$ & + & + & - & + \\
\hline S. G. 60 & $M$ & $\begin{array}{l}\text { Incompl. } \\
\text { Sub TH5 }\end{array}$ & 2 Months & 60 & + & $\neq$ & + & + & - & + \\
\hline
\end{tabular}




\section{Results}

The results obtained in 29 adults with neurogenic bladder disturbance due to incomplete traumatic cord lesions are presented under the following headings: development or alteration of bladder sensation, of detrusor contraction, the achievement of bladder control and the efficiency of micturition as shown by the decrease of residual urine.

The method is very helpful in the rehabilitation of micturition in patients with incomplete traumatic spinal cord lesions: 26 out of 29 patients gained perfect bladder sensation, 25 of them achieved satisfactory bladder contractions, 28 ended the stimulation programme with residual urine below $50 \mathrm{cc}$ and 17 out of 30 gained perfect bladder control, ten others became at least socially dry without need for pads and urinals.

Ten patients have subsequently recorded some weakening of the stimulation effect after I year of treatment and five of these have asked for re-stimulation.

\section{Discussion}

In discussing the results in patients with a paralysed bladder due to incomplete spinal lesions it can be argued that improvement in function could have occurred without this stimulation. This series does not have a control group treated without stimulation, but, as each of these patients shows a specific pattern of lesion with an individual capacity of recovery, such a control group would be rather difficult to obtain.

However, there are several points supporting the belief that it is really the stimulation and not the passage of time which brings the improvement in function. There is a close correlation between the recovery of detrusor function and the stimulation programme; a progress, which does not correlate with the recovery of other autonomic or somatic functions. The weakening of the stimulation effect after 6 to 12 months in some patients, who therefore require restimulation, also supports a causal relationship between stimulation and benefit.

\section{SUMMARY}

The effect of direct transurethral electrostimulation of the saline-filled bladder in 29 patients with bladder dysfunction after incomplete spinal cord injury is described. Following investigation by cystourethrography and cystomanometry, treatment was started from I4 days to 8 months after injury (average 3 months after injury).

The method was considered to be very helpful in the rehabilitation of micturition. Twenty-six out of 29 patients gained normal bladder sensation, 25 achieved satisfactory detrusor contractions and 29 had low residual urines. Seventeen patients developed perfect bladder control and ten more were socially dry without the need for appliances.

\section{RESUMÉ}

On decrit l'effet de la stimulation éléctrique transurethral directe de la vessie pleine de saline chez 29 malades avec une dysfonction de la vessie après une lésion incomplète de la colonne vertébrale. Après les investigations avec cystourethographie et cystomanometrie le traîtement avait commencé après quinze jours jusqu'à huit mois après la lésion (en moyenne trois mois, après la lésion). 
La méthode avait été trés secourable pour la rehabilitation de la miction. 26 de 29 malades ont obtenú des sensations normales dans leur vessie. 25 malades ont obtenú des contractions detrusoriales satisfaisantes et ont eu des urines residuales modestes. I7 malades ont developpé une contrôle parfaite sur la vessie et dix âutres étaient 'sec social' sans moyen.

\section{ZUSAMMENFASSUNG}

Bei 29 Patienten mit neurogener Blasenentleerungsstörung nach inkompletter Querschnittslähmung wurde eine meist mehrmonatige transurethrale Elektrostimulation der mit Elektrolytlösung $(\mathrm{NaCl} 0,9 \%$ ig) gefüllten Blase durchgeführt. Die Therapie wurde durchschnittlich 3 Monate nach dem Unfall begonnen, das kürzeste Intervall zwischen Unfall und Stimulationsbeginn betrug I4 Tage, das längste 8 Monate. Vor, während und nach der Behandlung wurde die Funktion der Blase und des Blasenauslaß urodynamisch untersucht bzw. kontrolliert.

Die Ergebnisse zeigen, daß die Methode wesentlich zur Rehabilitation der Blase beitragen kann: 26 der 29 Patienten erreichten wiederum eine normale Blasensensibilität, 25 eine ausreichende Detrusorkontraktion, 28 von 29 entleerten die Blase mit Restharnmengen unter $50 \mathrm{ml}$. I 7 von 29 hatten nach Beendigung der Stimulationsbehandlung eine gute Blasenkontrolle, weitere Io wurden zumindest 'socially dry' und benötigten keine Behelfe wie Urinal oder Windeln.

\section{REFERENCES}

Berger, D., Berger, K. \& Genton, W. (I978). Endovesical transurethral electrostimulation in the rehabilitation of neurogenic bladder in children. Eur. Urol., 4, 33-45.

FARNSWORTH, R. H. (I976). Electrostimulation in the management of neurogenic bladder disorders. Brit. F. Urol., 48, I49-150.

James, E. D. \& Flack, F. C. (I974). Assessment of recording nappy. Urol. int., 29, I 74-I75.

JANNECK, C. (1975). Electric stimulation of the bladder and the anal sphincter. A new way to treat the neurogenic bladder. Prog. paediatr. Surg., 9, II9-I39.

Katona, F., BenYo, L. \& LANG, J. (I 959). Über intraluminäre Elektrotherapie vor verschiedenen paralytischen Zuständen des gastrointestinalen Traktes mit Quadrangulärstrom. Z Zbl. Chir., 84, 929-933.

Katona, F. (1975). Stages of vegetative afferentation in reorganisation of bladder control during electrotherapy. Urol. int., 30, 192-203.

MADERSBACHER, H. (I978). Der Effekt der transurethralen Elektrostimulation auf die gelähmte und inkontinente Blase: objektive Ergebnisse. Urologe A, 17, 355-357.

MADERSBACHER, H. (I977). Combined pressure, flow, EMG and X-ray studies for the evaluation of neurogenic bladder disturbance: technique and results. Urol. Inter., 32, I76-I 83 .

Nicolas, J. L. \& Eckstein, H. B. (I975). Endovesical electrotherapy in treatment of urinary incontinence in spina-bifida patients. Lancet, I276-1277.

Seiferth, J., Larkamp, H. \& Heising, J. (I978). Erfahrungen mit der temporären, intravesicalen Elektrostimulation bei neurogener Blasenentleerungsstörung von Spinabifida-Kindern. Urologe A, 17, 353-357. 